\title{
Epilogue
}

\section{Multiple Voices and Future Directions in Jain Bioethics}

In this book we have explored the "cumulative tradition" of Jainism by mapping multiple Jain principles for, and perspectives about, contemporary bioethics. Our aim was to pay close attention to the diversity of Jain voices as an essential task in avoiding the perception that Jainism is a homogeneous tradition that speaks with a single voice. To this end, we outlined the development of the doctrines in textual sources over time, and highlighted the distinction between mendicants and laity, sectarian perspectives, and Jains living in India and in the diaspora.

In part 1 we strove to identify textually supported principles that would represent the complexity of Jainism as a self-contained, alternative philosophical worldview, with its own internal logic. We focused primarily on its evolving metaphysical, ethical, and soteriological frameworks as well as its rich history of encounters with medicine, which are conceptual areas that we found to be most relevant for bioethical discussions.

In part 2 we drew from traditional textual sources and contemporary Jain voices to explore various approaches to specific bioethical issues. While there is no direct line between Jain philosophy as expounded in the traditional texts and modern practice for many of the bioethical issues that we examined in the book, contemporary Jains have thought about most of the issues either personally, professionally, or academically. The survey that we conducted with medical professionals was particularly significant in helping us identify principles of application that reflect how contemporary Jains strive to bring together their Jain identity on one hand and the competing scientific values and ethical sensibilities of their broader cultural and social contexts on the other. Examining these contemporary approaches through a historical perspective allowed us to indicate lines of continuity and discontinuity between earlier and contemporary debates and dilemmas. 
We hope that our book will provide a foundation for future studies in the scarcely researched field of Jain bioethics. Particularly exciting areas of possible research include lay approaches to bioethical issues beyond Jain medical professionals and academics, contemporary mendicants and medicine, Jain bioethics in conversation with the views of other South Asian religious traditions, and indepth comparative studies of Jain bioethical viewpoints and Western normative ethics on the various issues that we covered in the book. Other salient research topics might include the wide social and ecological arenas of human-animal-plant relations and emerging technologies. Our research shows that Jain positions on bioethical topics do not easily match any of the predominant normative ethics theories, but further cross-cultural work needs to be done in this area to explore where the Jain voice fits into the bioethical debates and what its potential contributions to contemporary discussions are. Our work also opens up questions about representation: What do we mean when we speak of a "Jain" approach, and who represents "Jain" (bio)ethics? Combining textual studies with fieldwork could be particularly fruitful for the future study of Jain bioethics in this regard.

Our intention was to show that Jainism can be in a meaningful conversation with contemporary bioethics. In the beginning of the book, we pointed out the roots of Western bioethics, which are-in the call for an expansive understanding of life and the notion of a moral subject-reminiscent, at least in certain respects, of Jainism (and were perhaps even influenced by it). Our book traces a long history of practical ideals and pragmatic accommodations that developed in following such a wide-ranging metaphysics of life and corresponding ethical guidelines. We highlighted the personal, professional, and public conflicts and commitments that continue to interconnect in the Jain encounters with the issues of birth, life, death, illness, health, liberation, and well-being. Ethical duties toward one's jivva and the approaches to one's body ensuing from those duties are not stricly individualized. Rather, these aspects of Jain practice are informed by broader communal concerns and, as such, provide a foundation for addressing bioethical issues in their personal and public dimensions.

We strove to write a book that was not prescriptive in character. Rather than developing a model of what Jain bioethics should look like, we outlined and analyzed the foundations and applications that are already present in the tradition. Bioethics is not an alien discipline to Jainism, and issues related to it continuously arise in clinical settings. We were interested in learning how Jains have navigated what we today understand as bioethical concerns and to what extent, and how, their responses have drawn from their philosophical-religious background. It is our hope that this work has done justice to the rich tradition of Jainism, the everemerging field of bioethics, and the multiplicity of insistent lives with whom we share this experience of existence. 